\title{
Comparison of Two Quantitative Analysis Techniques to Predict the Evaluation of Product Form Design
}

\author{
Hung-Yuan Chen, ${ }^{1}$ Yu-Ming Chang, ${ }^{2}$ and Ting-Chun Tung ${ }^{1}$ \\ ${ }^{1}$ Department of Visual Communication Design, Southern Taiwan University of Science and Technology, Tainan 71005, Taiwan \\ ${ }^{2}$ Department of Creative Product Design, Southern Taiwan University of Science and Technology, Tainan 71005, Taiwan
}

Correspondence should be addressed to Hung-Yuan Chen; hungyuan@mail.stust.edu.tw

Received 12 June 2014; Accepted 4 September 2014; Published 21 September 2014

Academic Editor: Teen-Hang Meen

Copyright (c) 2014 Hung-Yuan Chen et al. This is an open access article distributed under the Creative Commons Attribution License, which permits unrestricted use, distribution, and reproduction in any medium, provided the original work is properly cited.

Consumer satisfaction with a product's form plays an essential role in determining the likelihood of its commercial success. A consumer perception-centered design approach is proposed in this study to aid product designers with incorporating consumers' perceptions of product forms in the design process. The consumer perception-centered design approach uses the linear modeling technique (multiple linear regression) and the nonlinear modeling technique (neural network) to determine the satisfying product form design for matching a given product image. A series of experimental evaluations are conducted to collect evaluation results for examining the relationship between the automobile profile features and the consumers' perceptions of the automobile image. The result of predictive performance comparison shows that both the nonlinear neural network modeling technique and the multiple linear regression technique are comparably good for predicting the consumers' likely response to a particular automobile profile since the predictive performance difference between the two modeling techniques is very slight in this study. Although this study has chosen a 2D automobile profile for illustration purposes, the concept of the proposed approach is expansively applicable to 3D automotive form design or other consumer product forms.

\section{Introduction}

Consumers interact with a huge number of diverse products during the course of their daily lives and therefore subconsciously develop sophisticated product evaluation skills. A consumer's purchase decision is based not only on a product's functionality and fitness for use, but also on the psychological response induced by its physical appearance. This phenomenon is particularly apparent in the case of mature consumer products such as automobiles, mobile phone, tableware, and computer accessories. These mature consumer products can still sell well in the market place even when lacking advanced technological features and functionalities provided that their form design finds favor with the consumers. As a result, consumer satisfaction with a product's form plays an essential role in determining the likelihood of its commercial success. However, product form design activities are often reduced to a discussion based on the designers' opinions and personal subjectivities, with no theoretical basis. To avoid the subjective judgments in the design process and to objectively relate consumers' psychological satisfaction with a product to its form features, many systematic design approaches have been proposed for modeling the correlation between the form features of a product and the consumers' perception of the product image [1-4]. Amongst such techniques, Kansei Engineering (KE) is a fundamental consumer-oriented systematic design approach in which the consumers' feelings or product image perceptions are expressed using suitable image descriptors. $\mathrm{KE}$ has emerged as one of the most powerful techniques for taking account of the correlation between a product's attributes and the induced product image during the design process [5].

The effectiveness of the KE approaches is crucially determined by the choice of analytical technique with which one can model the correlation between the product form and 
the corresponding consumer perception. The models used in the product design field to predict the likely consumer response to a particular product form are commonly based on either the conventional linear analysis techniques or the nonlinear modeling techniques (such as the artificial intelligent system). Conventional linear analysis techniques such as multiple linear regression (MLR) [6] and quantitative theory type I $[4,7]$ are commonly employed to interpret the relationships between the independent and dependent variables. MLR and quantitative theory type I are widely used because they are easy, are simple, and have good predictive performance. MLR is particularly good when the input data and output data relationship is linear. However, MLR and quantitative theory type I do not properly handle nonlinear relationships very well since the accuracy of the predicted results is seriously degraded if the independent and dependent variables are characterized by a nonlinear relationship. In contrast to conventional linear analysis techniques, nonlinear modeling techniques are defined as an emerging approach to learning the human mind in an uncertainty environment [8] and are free of the restriction on the type of relationship between the independent variables and dependent variables when constructing prediction models. Therefore, are the nonlinear modeling techniques suitable for exploring the relationship between the product form features and the corresponding consumers' perceptions? Or are the linear modeling techniques good enough to do so [9]? What kind of modeling technique should be used to predict the likely consumer response to a particular product form? To illustrate how the modeling techniques can be used to answer these research questions, this study considers the design of an automobile profile and explores the correlations between the design variables of automobile profile and the associated image perceptions using a linear analysis technique and a nonlinear modeling technique, respectively. This particular design case is chosen because the lateral contours of a vehicle are known to supply powerful stimuli influencing the consumer's image perceptions and the distinctiveness of a vehicle's outline is receiving increasing emphasis on manufacturers' marketing strategies nowadays.

Multiple linear regression (MLR) is a technique for modeling and analyzing numerical data consisting of the values of a large number of independent variables and a smaller number of dependent variables. MLR enables the identification of a set of independent variables which explain a certain proportion of the variance in a dependent variable at a specified significance level. The relationship between the independent variables and the dependent variables can be illustrated either graphically or, more usually, by means of an equation (model). Having established this model, it can be used to predict the value of the dependent variable for any given set of independent variables. Because of the effective learning and prediction capabilities for analyzing the relationship between the product form features (the input variables) and the consumers' perceptions (the output variables), nonlinear neural networks (NNs) have been successfully applied in a diverse range of fields $[10,11]$. NNs are well suited to formulate the product design process for matching the product form to the consumers' perceptions, which is often a black box and cannot be precisely described $[10,12,13]$.

From the discussions above, a consumer perceptioncentered design approach is proposed for modeling the correlation between the profile features of an automobile and the consumers' perception of the image projected by the automobile. Consumers' perceptions of the automobile profile image are described using single adjectives and a coordinatebased definition is used to define the automobile profile. In order to gather the evaluation data of consumers, the consumers' perception evaluations of automobile image are conducted. The prediction models of consumers' perceptions induced by the automobile profile images are constructed using MLR and NNs. The subsequent sections of this study are organized as follows. Section 2 presents a review of MLR and NNs. Section 3 presents the research implementation. Section 4 analyzes the results of constructing prediction models. Section 5 verifies the performance of prediction models and provides the predictive performance comparison between MLR models and NN models. Section 6 offers some brief conclusions.

\section{Review of Quantitative Analysis Techniques}

In this section, we present a brief outline of the relevant theories and algorithms, including the MLR and the NNs. These techniques are used to examine the relationship between the automobile profiles and the corresponding product images in this study.

2.1. Multiple Linear Regression (MLR). MLR is a traditional statistical technique for modeling the linear relationships between the input variables (i.e., the independent variables) $x_{i} \in R^{n}$ and the desired output variables (i.e., the dependent variables) $y_{i} \in R$. The regression equation for an MLR problem involving $l$ data samples has the form

$$
y_{i}=b_{0}+b_{1} x_{i, 1}+\cdots+b_{n} x_{i, n}+e_{i}, \quad i=1, \ldots, l,
$$

where $b_{0}$ is the regression constant, $b_{1}, \ldots, b_{n}$ are the partial regression coefficients corresponding to the $n$ input variables, and $e_{i}$ is the error term. The regression coefficients are generally estimated using an ordinary least squares (OLS) procedure such that the sum of the squared errors $\left(\sum_{i=1}^{l} e_{i}\right)$ is minimized. In MLR, the coefficient of determination, $R^{2}$, indicates the percentage of the variation in $y_{i}$ explained by the independent variables $x_{i}$. In other words, the value of $R^{2}$ (ranging from 0 to 1 ) indicates the goodness of fit of the regression model. The coefficients $b_{1}, \ldots, b_{n}$ in (10), commonly referred to as the unstandardized regression coefficients, can be used to construct the regression equation directly. However, the standardized regression coefficients, $\beta_{1}, \ldots, \beta_{n}$, provide a more suitable means of analyzing the relative importance of the different variables. Note that each standardized regression coefficient represents the change in response of appropriate per standard deviation change in one output value $y$.

Having established this equation (model), it can be used to predict the value of the dependent variable for any given 
set of independent variables. In addition, as described in the following discussions, MLR enables a screening of the independent variables such that the performance of the prediction model is enhanced. It is known that increasing the number of independent variables in a regression equation improves the fit to the training set but reduces the predictive ability of the model when supplied with a set of input data taken from outside the training set. Therefore, it is necessary to identify the subset of independent variables which collectively enhance the accuracy of the prediction model. Generally speaking, the optimal set of independent variables is compiled using one of three different methods, namely, the forward selection method, the backward elimination method, or the stepwise method [14]. Of these three methods, MLR with a stepwise procedure is particularly advantageous since it provides the combinatorial benefits of the forward selection method and the backward elimination method, respectively.

2.2. Neural Networks. NNs are nonlinear models and are widely used to examine the complex relationship between input variables and output variables. In this study, we use the multilayered feed-forward neural networks trained with the back-propagation learning algorithm, as it is an effective and the popular supervised learning algorithm. A typical threelayer network consists of an input layer, an output layer, and one hidden layer, with $n, m$, and $p$ neurons, respectively (indexed by $i, j$, and $k$, resp.) [15]. $w_{i j}$ and $w_{j k}$ represent the weights for the connection between neuron $i(i=1,2, \ldots, n)$ and neuron $j(j=1,2, \ldots, m)$ and between neuron $j(j=$ $1,2, \ldots, m)$ and neuron $k(k=1,2, \ldots, p)$, respectively. In training the network, a set of input patterns or signals, $\left(x_{1}, x_{2}, \ldots, x_{n}\right)$, is presented to the network input layer. The network then propagates the inputs from layer to layer until the output layer generates the outputs. This involves the generation of the outputs $\left(y_{j}\right)$ of the neurons in the hidden layer as given in (2) and the outputs $\left(y_{k}\right)$ of the neurons in the output layer as given in (3). One has

$$
\begin{aligned}
& y_{j}=f\left(\sum_{i=1}^{n} x_{i} w_{i j}-\theta_{i}\right), \\
& y_{k}=f\left(\sum_{i=1}^{m} x_{j} w_{j k}-\theta_{k}\right),
\end{aligned}
$$

where $f(\cdot)$ is the sigmoid activation function as given in (4) and ${ }_{-} j$ and $\_$are threshold values

$$
f(X)=\frac{1}{1+e^{-X}} .
$$

If the outputs $\left(y_{k}\right)$ generated by (3) are different from the target outputs $\left(y_{k}^{*}\right)$, errors $\left(e_{1}, e_{2}, \ldots, e_{p}\right)$ are calculated by (5) and then propagated backwards from the output layer to the input layer in order to update the weights for reducing the errors:

$$
e_{k}=y_{k}^{*}-y_{k}
$$

The weights $\left(w_{j k}\right)$ at the output neurons are updated as $w_{j k}+$ $\Delta w_{j k}$, where $\Delta w_{j k}$ is computed by (known as the delta rule)

$$
\Delta w_{j k}=\alpha y_{j} \delta_{k}
$$

where $\alpha$ is the learning rate (usually $0<\alpha \leq 1$ ) and $\delta_{k}$ is the error gradient at neuron $k$, given as

$$
\delta_{k}=y_{k}\left(1-y_{k}\right) e_{k}
$$

The weights $\left(w_{i j}\right)$ at the hidden neurons are updated as $w_{i j}+$ $\Delta w_{i j}$, where $\Delta w_{i j}$ is calculated by

$$
\Delta w_{i j}=\alpha x_{i} \delta_{j}
$$

where $\alpha$ is the learning rate (usually $0<\alpha \leq 1$ ) and $\delta_{j}$ is the error gradient at neuron $j$, given as

$$
\delta_{j}=y_{j}\left(1-y_{j}\right) \sum_{k=1}^{p} \delta_{k} w_{j k} .
$$

The training process is repeated until a specified error criterion is satisfied.

\section{Implementation Procedure and Steps}

3.1. Automobile Profile Definition and Evaluation Samples. This study collected a large number of pictures of commercial automobiles and the corresponding body length datum. These pictures were then examined to identify the various profile characteristics which collectively define the overall automobile profile based on a human interpretation of the distinctive component features of that particular profile. A total of 144 vehicle pictures with a side-view orientation were collected from automobile magazines, catalogs, and websites. The 144 automobile images were then converted into profile samples by tracing their contour features with 16 Bézier segments using computer graphic software.

Table 1 shows a typical example of a car profile constructed using the Bézier segments. Of the 16 Bézier segments $\left(C_{1} \sim C_{16}\right)$ in the profile, the chassis segment $\left(C_{15}\right)$ was assumed to be a straight line with just two control points since this profile feature is approximately straight in most automobiles. However, the remaining segments were each assigned four control points to enable the introduction of detailed profile variations in each segment. As stated above, each profile was constructed using 16 Bézier segments. As a result, a total of 16 control points were located at the joints between contiguous Bézier curves. Since these joints were used by both segments when tracing the automobile profile, the overall car profile was defined using a total of 46 control points (i.e., $2+(15 \times 4)-16=46)$. In order to define the automobile profile, a coordinate-based definition approach was conducted in this study. Control point $P_{1}$ was specified as the origin $(0,0)$ and the coordinate positions of each of the other control points were then recorded with respect to this origin point. Since each control point was assigned both $X$ and $Y$-axis coordinates, the automobile profile was defined using a total of 92 (i.e., $46 \times 2$ ) coordinate data items. Further, 
TABLE 1: Tracing of automobile profile using 16 Bézier curves.

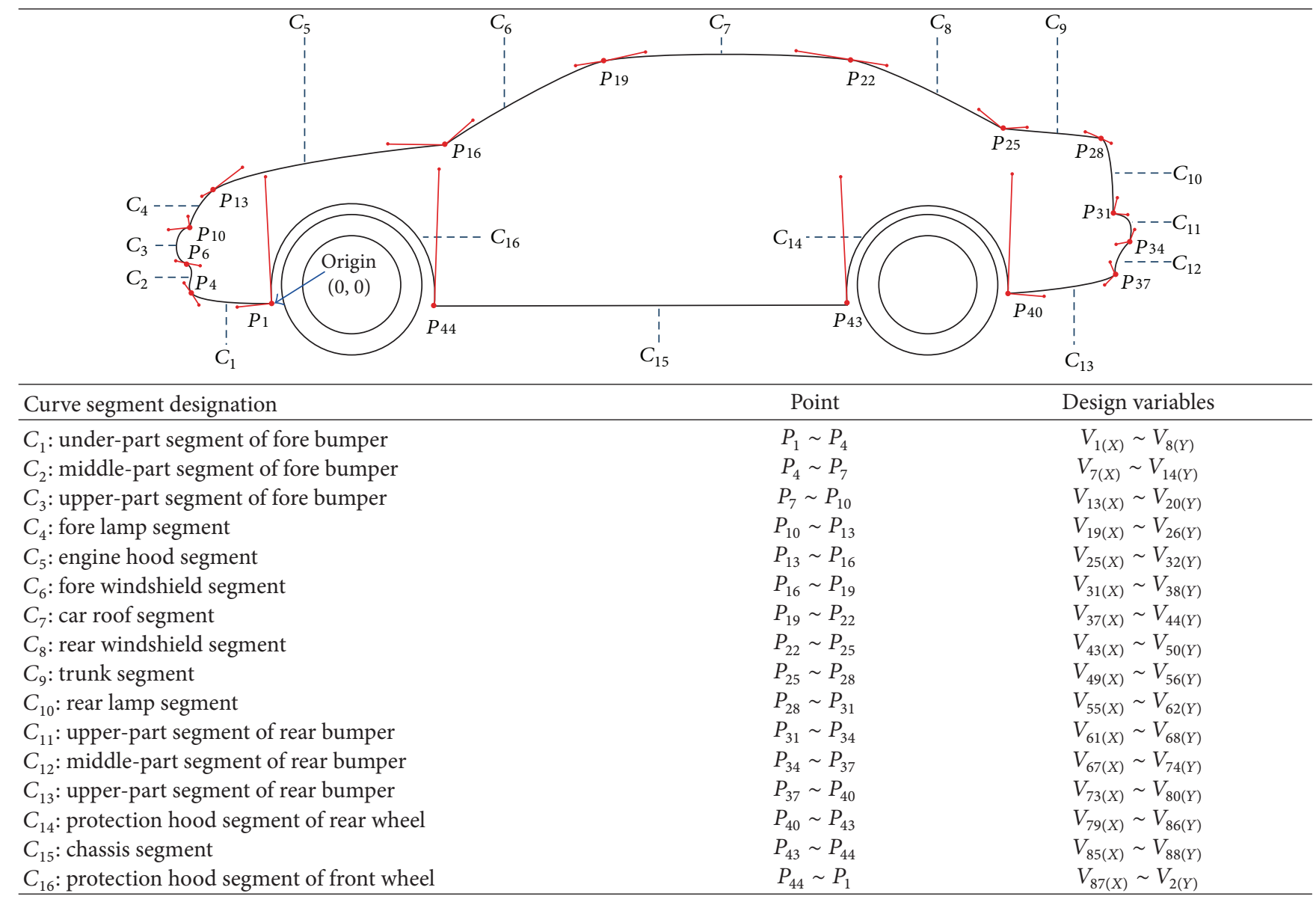

each automobile profile sample was painted $50 \%$ gray in order to enhance its visual impact and was uniformly reduced in size using a scaling factor of $1: 25$ based on actual automobile body length to individually display on an A4-sized card. The total set of 144 automobile profile samples were considered in the subsequent evaluation trials.

3.2. Selecting the Representative Image Descriptors of Automobile Profile. Consumers commonly use image words (i.e., adjectives) to express their image perceptions of a product. Although many different image words are used when describing everyday products, the image words applicable to an automobile profile are more limited. In this study, 3 designers and 3 white-collar individuals from nondesign backgrounds were invited to participate in a discussion aimed at identifying suitable descriptors with which one can describe the possible psychological responses of a consumer when presented with the sample automobile profiles. The image descriptors were elicited from the participants using the following four-step procedure.

Step 1. The 144 automobile profiles were reviewed, and the image words used by the individual participants to describe their perceptions of the image projected by each automobile profile were recorded.
TABLE 2: Selection and classification of image descriptors.

\begin{tabular}{ll}
\hline $\begin{array}{l}\text { Image } \\
\text { descriptors }\end{array}$ & Image words contained \\
\hline Modern & $\leftarrow$ Modern, Advanced, Technical, Novel, Fashionable \\
Formal & $\begin{array}{l}\text { Formal, Popular, Robust, Geometric, Rational, } \\
\text { Mature }\end{array}$ \\
Classical & $\leftarrow$ Classical, Sleek, Stylish, Smooth \\
Rakish & $\begin{array}{l}\text { Rakish, Personal, Dashing, Young, Peculiar, } \\
\text { Speedy, Future, Streamlined, Wild }\end{array}$ \\
Elegant & $\leftarrow \begin{array}{l}\text { Elegant, Gorgeous, Luxurious, Noble, Lofty, } \\
\text { Gentle }\end{array}$ \\
Family & $\leftarrow$ Family, Leisure, Vital \\
\hline
\end{tabular}

Step 2. The focus group method [16] was applied to select 33 suitable product image descriptors for a generic automobile profile.

Step 3. The Kawakita Jiro (K J) method [17] was then applied to classify the 33 image words in accordance with their semantic similarities. As shown in Table 2, six basic descriptor groups were identified.

Step 4. From each group, one image descriptor was chosen to represent the overall characteristics of the group, that is, Modern, Formal, Classical, Rakish, Elegant, and Family. 
TABLE 3: Results of MLR analysis in six image perception domains.

\begin{tabular}{|c|c|c|}
\hline MLR prediction models & $R$ & $R^{2}$ \\
\hline $\begin{array}{l}\text { "Rakish" image } \\
=-0.123(v .3 x)-0.138(v .9 x)+0194(v .17 x)-0.549(v .19 x)+0.400(v .21 x)+0.182(v .31 x) \\
\quad+0.171(v .39 x)+0.112(v .42 y)-0.107(v .43 x)-0.523(v .44 y)+0.105(v .49 x)+0.377(v .50 y) \\
\quad-0.087(v .58 y)+0.162(v .60 y)-0.184(v .67 x)-0.060(v .73 x)-0.335(v .88 y)+0.263(v .89 x) \\
\quad+4.450\end{array}$ & 0.917 & 0.819 \\
\hline $\begin{array}{l}\text { "Family" image } \\
=0.067(v .3 x)-0.098(v .5 x)+0.092(v .9 x)+0.099(v .11 x)+0.338(v .19 x)-0.382(v .21 x) \\
\quad-0.313(v .30 y)-0.286(v .38 y)+0.098(v .42 y)+0.541(v .44 y)+0.145(v .48 y)+0.053(v .51 x) \\
\quad+0.204(v .58 y)-0.265(v .76 y)-0.203(v .82 y)+5.804\end{array}$ & 0.840 & 0.671 \\
\hline $\begin{array}{l}\text { "Formal" image } \\
=0.075(v .7 x)-0.037(v .15 x)-0.078(v .17 x)-0.390(v .32 y)+0.098(v .33 x)+0.386(v .36 y) \\
\quad-0.113(v .37 x)+0.095(v .56 y)+0.041(v .57 x)-0.124(v .63 x)+0.112(v .68 y)+0.209(v .69 x) \\
\quad-0.279(v .70 y)+0.146(v .78 y)-0.165(v .87 x)-0.266(v .92 y)+5.435\end{array}$ & 0.849 & 0.685 \\
\hline $\begin{array}{l}\text { "Classical" image } \\
=-0.043(v .7 x)+0.128(v .9 x)-0.127(v .10 y)-0.038(v .15 x)-0.094(v .23 x)+0.101(v .33 x) \\
\quad-0.212(v .34 y)+0.195(v .36 y)-0.140(v .37 x)+0.084(v .41 x)+0.171(v .48 y)-0.140(v .56 y) \\
\quad+0.022(v .59 x)+0.040(v .73 x)-0.039(v .77 x)-0.085(v .92 y)+5.208\end{array}$ & 0.904 & 0.791 \\
\hline $\begin{array}{l}\text { "Elegant" image } \\
=-0.067(v .3 x)-0.228(v .18 y)+0.522(v .20 y)-0.308(v .22 y)-0.075(v .25 x)+0.364(v .44 y) \\
\quad-0.046(v .47 x)+0.121(v .71 x)-0.214(v .76 y)+5.259\end{array}$ & 0.813 & 0.639 \\
\hline $\begin{array}{l}\text { "Modern" image } \\
=0.050(v .5 x)-0.128(v .9 x)-0.201(v .10 y)+0.165(v .12 y)+0.100(v .25 x)+0.160(v .39 x) \\
\quad-0.072(v .41 x)+0.072(v .42 y)-0.120(v .43 x)-0.517(v .44 y)+0.202(v .48 y)+0.103(v .49 x) \\
\quad-0.157(v .57 x)+0.193(v .61 x)+0.174(v .62 y)-0.060(v .73 x)-0.151(v .84 y)+0.217(v .92 y) \\
+4.304\end{array}$ & 0.836 & 0.661 \\
\hline
\end{tabular}

3.3. Evaluation of Automobile Profile Images. The 144 automobile profile samples and the six image descriptors were used to perform an examination of the correlation between the automobile profiles and their associated image perceptions. The investigation was performed by 32 subjects. In the evaluation process, each profile image was assessed in terms of the six image descriptors. Note that the evaluation method was performed separately by each of the 32 subjects. Taking the first image descriptor, each subject divided the 144 automobile profile samples into 3 groups, that is, low (L), medium (M), and high ( $\mathrm{H})$, in accordance with his or her intuitive perception of the extent to which the image descriptor described the feelings induced by the profile. The subject then further divided each of the 3 groups into 3 subgroups. In this way, the 144 automobile profile samples were divided into a total of 9 groups. Each automobile profile was then assigned a score from 1 to 9 (LL to $\mathrm{HH}$ ) depending on the group to which it was assigned. Having classified the 144 profiles in terms of the first image descriptor, the twostage classification process was repeated for each of the other five image descriptors. Finally, the evaluation results obtained from the 32 subjects for the 144 automobile profile samples were used to analyze the relationship between the automobile profile features and the associated image perceptions.

\section{Constructing Prediction Models of Automobile Profile Image}

4.1. MLR Prediction Models. MLR with a stepwise selection procedure was applied to the evaluation data obtained for the automobile profiles in order to construct functional relationships between the design variables of the automobile profile and the corresponding consumer responses in each of the image perception domains. The MLR models were constructed subject to the criteria that a $P$ value of less than 0.1 was required for entry to the model such that only those design variables having a significant effect on the consumers' perception of the automobile profile were retained. In performing the MLR analyses, the independent variables corresponded to the 92 design variables of automobile profile and the six dependent variables were specified as the mean value of the evaluation scores assigned in the corresponding image perception domain.

Overall, Table 3 shows the functional models relating the design variables of the automobile profile to an evaluative rating in each of the six product image perception domains. In this table, the multiple correlation coefficient $R$ varies from 0.813 (Elegant) to 0.917 (Rakish) while the adjusted $R^{2}$ values vary from 0.639 to 0.819 , respectively. This result implies the existence of a significant relationship between the 92 design variables and the six image perception domains.

4.2. NN Prediction Models. In constructing and training the NN used to predict the relationship between the 144 automobile profiles and the corresponding image descriptors, the 92 design variables for each automobile profile were used as the input neurons of the $\mathrm{NN}$ and the average values of the 6 image evaluations were used as the target values of the output neurons. In attempting to establish the optimal NN model, three different models were constructed, 
TABLE 4: RMSE of NN prediction models for automobile profile training dataset.

\begin{tabular}{|c|c|c|c|}
\hline $\begin{array}{l}\text { Number of } \\
\text { training } \\
\text { epochs }\end{array}$ & $\begin{array}{l}\text { NN-Arith. } \\
\text { I: } 92 \text { neurons } \\
\text { H: } 49 \text { neurons } \\
\text { O: } 6 \text { neurons }\end{array}$ & $\begin{array}{l}\text { NN-Geom. } \\
\text { I: } 92 \text { neurons } \\
\text { H: } 23 \text { neurons } \\
\text { O: } 6 \text { neurons }\end{array}$ & $\begin{array}{l}\text { NN-Sum } \\
\text { I: } 92 \text { neurons } \\
\text { H: } 98 \text { neurons } \\
\text { O: } 6 \text { neurons }\end{array}$ \\
\hline 1000 & 0.1028 & 0.1031 & 0.1069 \\
\hline 2000 & 0.0956 & 0.0970 & 0.0964 \\
\hline 5000 & 0.0846 & 0.0871 & 0.0872 \\
\hline 10,000 & 0.0775 & 0.0787 & 0.0791 \\
\hline 20,000 & 0.0672 & 0.0685 & 0.0684 \\
\hline 30,000 & 0.0620 & 0.0638 & 0.0643 \\
\hline 40,000 & 0.0588 & 0.0606 & 0.0610 \\
\hline 50,000 & 0.0576 & 0.0599 & 0.0603 \\
\hline 60,000 & 0.0567 & 0.0587 & 0.0592 \\
\hline 70,000 & 0.0558 & 0.0577 & 0.0584 \\
\hline 80,000 & 0.0553 & 0.0570 & 0.0579 \\
\hline 90,000 & 0.0550 & 0.0570 & 0.0577 \\
\hline
\end{tabular}

"I," "H," and "O" indicate the input, hidden, and output layer, respectively.

differing only in terms of the number of neurons within their single hidden layer. The number of neurons in the hidden layer was specified in accordance with three common rules, namely, the arithmetic mean (Arith.), the geometric mean (Geom.), and the sum (Sum) of the input and output neurons, respectively. As stated above, the 144 automobile profiles and their associated product images were defined in terms of the 92 coordinate variables and 6 product image descriptors, respectively. Consequently, each of the three NN models comprised 92 input neurons and 6 output neurons. In accordance with the three rules specified above, the hidden layers of the three models therefore comprised 49 (Arith), 23 (Geom.), and 98 (Sum) neurons, respectively. The three NN models were trained using the coordinate-based definition data associated with the 144 automobile profile samples and the corresponding image perception values obtained from the evaluation trials. The three NN models were trained using a sigmoid transformation function and a delta-rule learning rule, respectively.

As shown in Table 4, all three models converged rapidly to an RMSE value of just over 0.1 after 1000 training epochs and to a value of just less than 0.1 after approximately 2000 epochs. These results indicate that the NN models all successfully converged as the number of training epochs increased. The three NN models were trained continuously for a total of 90,000 epochs. As shown in Table 4, the NN-Arith. model obtained its best predictive performance after 90,000 epochs (RMSE $=0.0550$ ), while the NN-Geom and NN-Sum models obtained their best performances after 80,000 epochs $($ RMSE $=0.0570)$ and 90,000 epochs $($ RMSE $=0.0577)$, respectively. Although the difference between the RMSE values of the three NN models is less than 0.0030 (0.0577 $0.0550=0.0027)$, the RMSE value of the NN-Arith. model is lower (i.e., better) than those of the other two models after 90,000 training epochs. Consequently, the NN-Arith. model was adopted as the operational NN model for predicting the values of each of the six automobile image descriptors for any given automobile profile definition data.

\section{Validation and Comparison of Prediction Model Performance}

5.1. Performance Evaluation of Prediction Models. To verify the predictive ability of the MLR and NN models, six new automobile profiles were designed using the respective coordinate-based definitions. The six automobile profiles were displayed on individual A4-sized cards and were evaluated by a group of 30 subjects using nine-point Likert scales. The product image perception in each of the six domains associated with the automobile profile was also predicted by substituting the relevant design variable values of each verification sample into the functional models. The discrepancy between the Likert scale evaluation scores assigned to each verification sample by the 30 subjects and those predicted by the functional models was then assessed using the following root-mean-square-error index (EI):

$$
\mathrm{EI}=\sqrt{\frac{\sum_{i=1}^{n}\left(x_{i}-x_{o}\right)^{2}}{n}}
$$

where $x_{i}$ is the Likert scale point assigned by the $i$ th subject, $x_{o}$ is the Likert scale point predicted by the corresponding model, and $n$ is the number of participants involved in the validation experiments. The EI value in (10) gives the average difference between the evaluations of the subjects and those of the corresponding model, respectively, for a single point on the nine-point Likert scale. To enable the reliability of the prediction models to be more conveniently compared, a normalized EI value was obtained by dividing the result obtained from (10) by nine to yield an error rate (ER) in the interval $[0,1]$. Clearly, a lower value of ER indicates an enhanced predictive capability.

Tables 5 and 6 present the predictions, EI values, and corresponding error rate (ER) values of the MLR and NN models, respectively, for the six image perception domains associated with the six automobile verification examples (see Figure 1). From inspection, it is determined that the average ER varies from $13.32 \%$ for the "Family" image to $20.57 \%$ for the "Modern" image when evaluated using the MLR prediction models (see Table 5). By contrast, the average ER is found to vary from $13.38 \%$ for the "Family" image to $19.22 \%$ for the "Classical" image when evaluated using the NN prediction model (see Table 6). Overall, the results demonstrate the basic reliability of the two functional models in predicting the image projected by an automobile profile in each of the six perception domains.

\subsection{Prediction Performance Comparison between MLR Model} and NN Model. Table 7 summarizes the average ER values of the two functional models when applied to predict the consumer response to the automobile profile in each of the corresponding image perception domains. The only exception to this tendency occurs in the "Family" and 
TABLE 5: Performance evaluation of MLR prediction models.

\begin{tabular}{|c|c|c|c|c|c|c|}
\hline & Mean & Prediction & $\mathrm{ER}(\mathrm{EI} / 9)$ & Mean & Prediction & $\mathrm{ER}(\mathrm{EI} / 9)$ \\
\hline \multicolumn{4}{|c|}{ Rakish } & \multicolumn{3}{|c|}{ Family } \\
\hline 1 & 5.23 & 5.10 & $12.8 \%$ & 7.70 & 6.59 & $17.6 \%$ \\
\hline 2 & 2.77 & 2.88 & $12.8 \%$ & 6.33 & 5.81 & $15.0 \%$ \\
\hline 3 & 5.23 & 4.99 & $17.1 \%$ & 7.57 & 7.20 & $11.1 \%$ \\
\hline 4 & 2.33 & 2.49 & $14.3 \%$ & 8.43 & 8.49 & $8.0 \%$ \\
\hline 5 & 1.77 & 4.56 & $31.9 \%$ & 6.23 & 6.56 & $13.6 \%$ \\
\hline \multirow[t]{3}{*}{6} & 6.80 & 7.08 & $13.3 \%$ & 6.37 & 5.97 & $14.6 \%$ \\
\hline & \multicolumn{3}{|c|}{ Average ER = 17.03\% } & \multicolumn{3}{|c|}{ Average ER = 13.32\% } \\
\hline & \multicolumn{3}{|c|}{ Formal } & \multicolumn{3}{|c|}{ Classical } \\
\hline 1 & 4.33 & 5.07 & $21.0 \%$ & 3.40 & 4.91 & $22.0 \%$ \\
\hline 2 & 6.63 & 5.42 & $21.6 \%$ & 5.53 & 5.72 & $13.2 \%$ \\
\hline 3 & 5.10 & 5.01 & $14.2 \%$ & 4.53 & 5.77 & $23.6 \%$ \\
\hline 4 & 6.47 & 6.41 & $15.4 \%$ & 5.40 & 4.74 & $18.0 \%$ \\
\hline 5 & 4.73 & 3.27 & $22.4 \%$ & 3.20 & 3.95 & $12.1 \%$ \\
\hline \multirow[t]{3}{*}{6} & 3.47 & 4.89 & $20.5 \%$ & 4.93 & 5.29 & $20.1 \%$ \\
\hline & \multicolumn{3}{|c|}{ Average ER = 19.18\% } & \multicolumn{3}{|c|}{ Average ER = 18.17\% } \\
\hline & \multicolumn{3}{|c|}{ Elegant } & \multicolumn{3}{|c|}{ Modern } \\
\hline 1 & 4.50 & 4.24 & $18.1 \%$ & 4.93 & 4.14 & $18.7 \%$ \\
\hline 2 & 8.10 & 6.63 & $19.0 \%$ & 3.97 & 4.10 & $20.2 \%$ \\
\hline 3 & 7.70 & 6.59 & $17.6 \%$ & 5.23 & 4.84 & $16.5 \%$ \\
\hline 4 & 5.17 & 7.29 & $27.1 \%$ & 4.60 & 3.91 & $21.0 \%$ \\
\hline 5 & 4.93 & 4.85 & $14.9 \%$ & 4.63 & 6.25 & $24.9 \%$ \\
\hline \multirow[t]{2}{*}{6} & 4.57 & 5.00 & $14.2 \%$ & 5.47 & 6.39 & $22.1 \%$ \\
\hline & \multicolumn{3}{|c|}{ Average $\mathrm{ER}=\mathbf{1 8 . 4 8 \%}$} & \multicolumn{3}{|c|}{ Average ER = 20.57\% } \\
\hline
\end{tabular}

TABLE 6: Performance evaluation of NN prediction model.

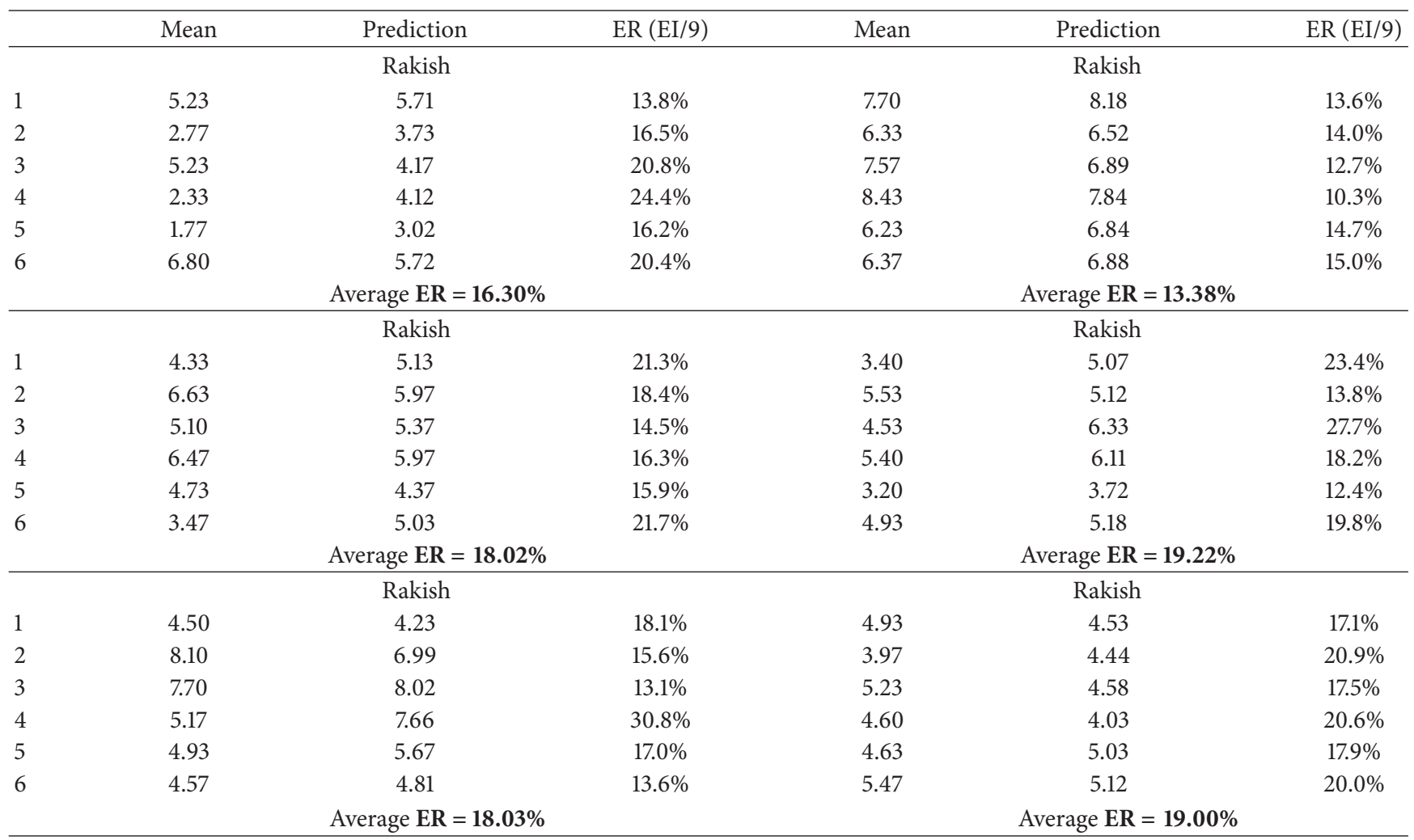




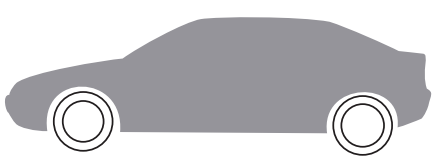

v.1

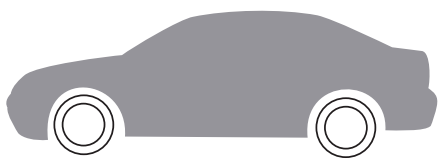

v.4

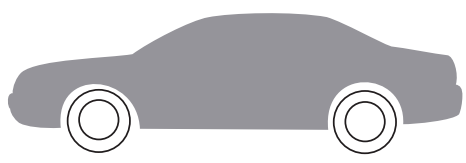

v.2

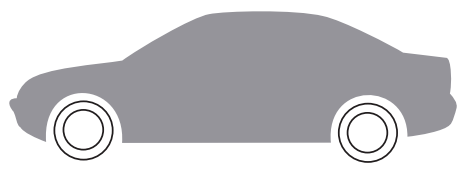

v.5

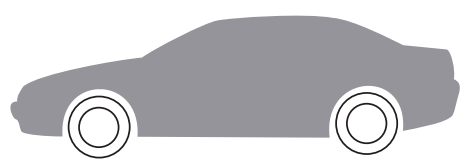

v.3

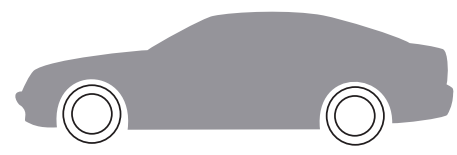

v.6

FIGURE 1: Automobile profiles used for functional model verification purposes.

the "Classical" image perception domain, in which the average ER value of the MLR model (Family = 13.32\%; Classical $=18.17 \%)$ is slightly lower than that of the NN model $($ Family $=13.38 \%$; Classical $=19.22 \%)$. Observing the mean ER data presented in the lower row of the table, the mean average ER values of the MLR model and the NN model for the automobile profile are $17.79 \%$ and $17.33 \%$, respectively. Comparing the mean average ER value of the MLR model with that of the NN model, it is observed that the latter is slightly lower (i.e., better) than the former. This finding seems to imply that the predictive performance of the NN model is slightly superior to that of the MLR model. However, while the inclusion of a large number of design variables enhances the efficiency of a general predictive model, it also increases the model complexity and therefore causes the designer problems in understanding the true nature of the relationship between the consumers' image perception and the individual design variables. Although the average ER values of the MLR model are slightly inferior to those of the NN, the MLR model has the advantage that it can use fewer design variables to predict the consumers' likely response to the automobile profile, and thus the functional relationships between the input design variables and the image perception values are not only more straightforward than those in the $\mathrm{NN}$ model but also more intuitively understandable. From inspection, the difference in the mean average ER values of the MLR and $\mathrm{NN}$ models is found to be less than $0.5 \%(17.79 \%-17.33 \%=$ $0.46 \%)$. Thus, overall, it can be inferred that the MLR model also represents a good solution for predicting the consumer's image perceptions of the automobile profile since it achieves a predictive ability very close to that of the NN model.

\section{Conclusions}

In this study, the relationships between the independent variables and the dependent variables are established using MLR and NN modeling techniques. The various models have all been verified by comparing the predicted consumers' perception of the product image in each product image domain with the corresponding manual evaluation results. Although the verification result presented in Table 7 has shown that the predictive performance of the NN model is slightly better than that of the MLR model, the difference in the predictive performance of the two models is very
TABLE 7: Comparison of average ER values of two prediction models.

\begin{tabular}{lcc}
\hline & $\begin{array}{c}\text { MLR prediction } \\
\text { Model }\end{array}$ & $\begin{array}{c}\text { NN prediction } \\
\text { Model }\end{array}$ \\
\hline Rakish & $17.03 \%$ & ${ }^{*} 16.30 \%$ \\
Family & ${ }^{*} 13.32 \%$ & $13.38 \%$ \\
Formal & $19.18 \%$ & ${ }^{*} 18.02 \%$ \\
Classical & ${ }^{*} 18.17 \%$ & $19.22 \%$ \\
Elegant & $18.48 \%$ & ${ }^{*} 18.03 \%$ \\
Modern & $20.57 \%$ & ${ }^{*} 19.00 \%$ \\
Mean of all average & $\mathbf{1 7 . 7 9 \%}$ & ${ }^{*} \mathbf{1 7 . 3 3} \%$ \\
ER & &
\end{tabular}

Asterisks indicate that average ER value of prediction model is lower (i.e., better).

slight here. Thus, this finding implies that the nonlinear NN modeling technique and the MLR technique are comparably good for predicting the consumers' likely response to a particular automobile profile. However, NN model with their sophisticated nonlinear algorithms is often opaque and it is therefore frequently difficult to recognize the specific design variables which dominate the consumers' response to the product design. By contrast, MLR technique allows designers to construct relationship models comprising only those independent variables which exert the most significant effect on the dependent values. Nevertheless, nonlinear relationship modeling using MLR technique results in poor predictive performance. In future study, it would be worthwhile considering the use of the integration model which combines the variable selection advantage of MLR and the sophisticated data analysis capabilities of NN for establishing the relationship between the dependent variables and the independent variables in the product form design field. Although this study has chosen a 2D automobile profile for illustration purposes, the concept of the proposed approach is expansively applicable to 3D automotive form design or other consumer product forms.

\section{Conflict of Interests}

The authors declare that there is no conflict of interests regarding the publication of this paper. 


\section{References}

[1] H. H. Lai, Y. C. Lin, C. H. Yeh, and C. H. Wei, "User-oriented design for the optimal combination on product design," International Journal of Production Economics, vol. 100, no. 2, pp. 253267, 2006.

[2] C. C. Chen and M. C. Chuang, "Integrating the Kano model into a robust design approach to enhance customer satisfaction with product design," International Journal of Production Economics, vol. 114, no. 2, pp. 667-681, 2008.

[3] K.-C. Wang, "A hybrid Kansei engineering design expert system based on grey system theory and support vector regression," Expert Systems with Applications, vol. 38, no. 7, pp. 8738-8750, 2011.

[4] Y.-C. Lin, C.-H. Yeh, C.-C. Wang, and C.-C. Wei, "Is the linear modeling technique good enough for optimal form design? A comparison of quantitative analysis models," The Scientific World Journal, vol. 2012, Article ID 689842, 13 pages, 2012.

[5] M. Nagamachi, "Kansei engineering as a powerful consumeroriented technology for product development," Applied Ergonomics, vol. 33, no. 3, pp. 289-294, 2002.

[6] Y.-M. Chang and H.-Y. Chen, "Application of novel numerical definition-based systematic approach (NDSA) to the design of knife forms," Journal of the Chinese Institute of Industrial Engineers, vol. 25, no. 2, pp. 148-161, 2008.

[7] Y. C. Lin, H. H. Lai, and C. H. Yeh, "Consumer-oriented product form design based on fuzzy logic:A case study of mobile phones," International Journal of Industrial Ergonomics, vol. 37, no. 6, pp. 531-543, 2007.

[8] P. T. Helo, Q. L. Xu, S. J. Kyllönen, and R. J. Jiao, "Integrated Vehicle Configuration System-Connecting the domains of mass customization," Computers in Industry, vol. 61, no. 1, pp. 44-52, 2010.

[9] R. B. Page and A. J. Stromberg, "Linear methods for analysis and quality control of relative expression ratios from quantitative real-time polymerase chain reaction experiments," The Scientific World Journal, vol. 11, pp. 1383-1393, 2011.

[10] H. H. Lai, Y. C. Lin, and C. H. Yeh, "Form design of product image using grey relational analysis and neural network models," Computers and Operations Research, vol. 32, no. 10, pp. 2689-2711, 2005.

[11] B. Kim, J. Lee, J. Jang, D. Han, and K.-H. Kim, "Prediction on the seasonal behavior of hydrogen sulfide using a neural network model," TheScientificWorldJournal, vol. 11, pp. 992-1004, 2011.

[12] S. Haykin, Neural Networks: A Comprehensive Foundation, Prentice Hall, Upper Saddle River, NJ, USA, 1999.

[13] Y. Kwon, O. A. Omitaomu, and G.-N. Wang, "Data mining approaches for modeling complex electronic circuit design activities," Computers and Industrial Engineering, vol. 54, no. 2, pp. 229-241, 2008.

[14] R. H. Myers, Classical and Modern Regression with Application, PWSKENT, Boston, Mass, USA, 2nd edition, 1990.

[15] M. Negnevitsky, ArtiIcial Intelligence, Addison-Wesley, New York, NY, USA, 2002.

[16] D. McDonagh, A. Bruseberg, and C. Haslam, "Visual product evaluation: exploring users' emotional relationships with products," Applied Ergonomics, vol. 33, no. 3, pp. 231-240, 2002.

[17] N. Cross, Engineering Design Methods: Strategies for Product Design, John Wiley \& Sons, Chichester, UK, 1994. 


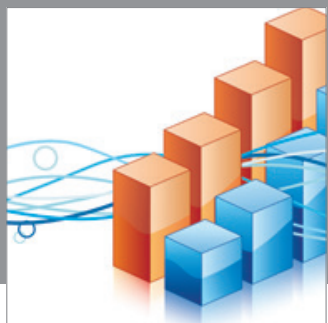

Advances in

Operations Research

mansans

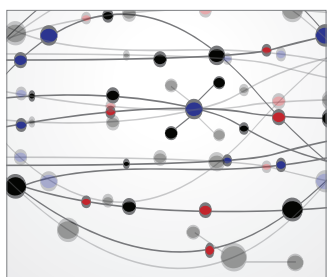

The Scientific World Journal
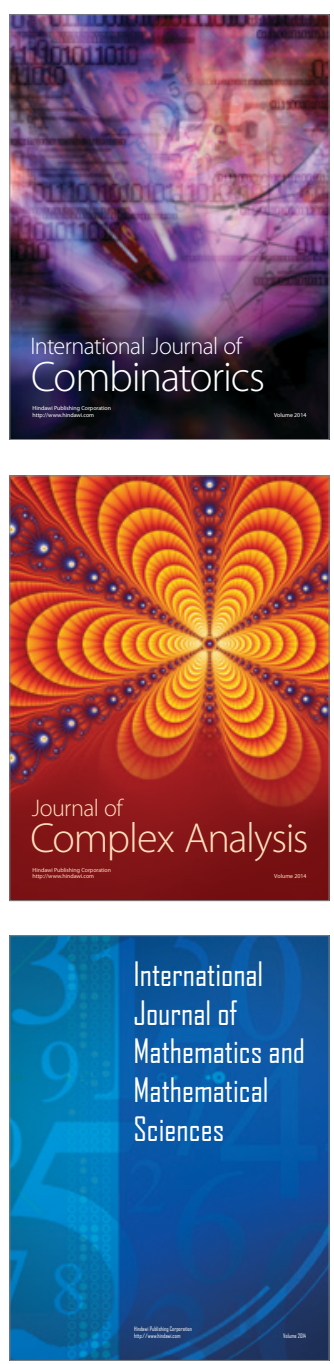
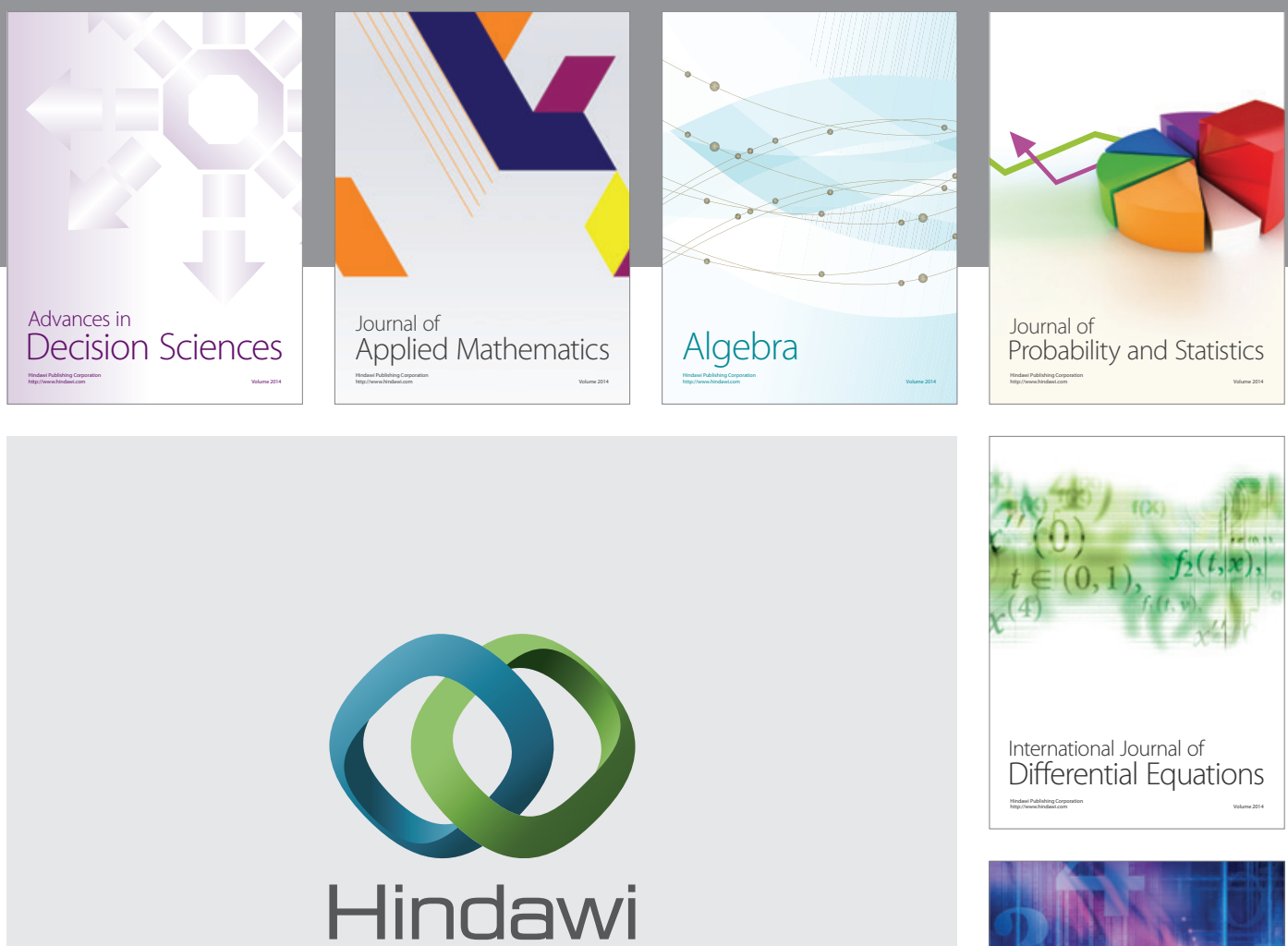

Submit your manuscripts at http://www.hindawi.com
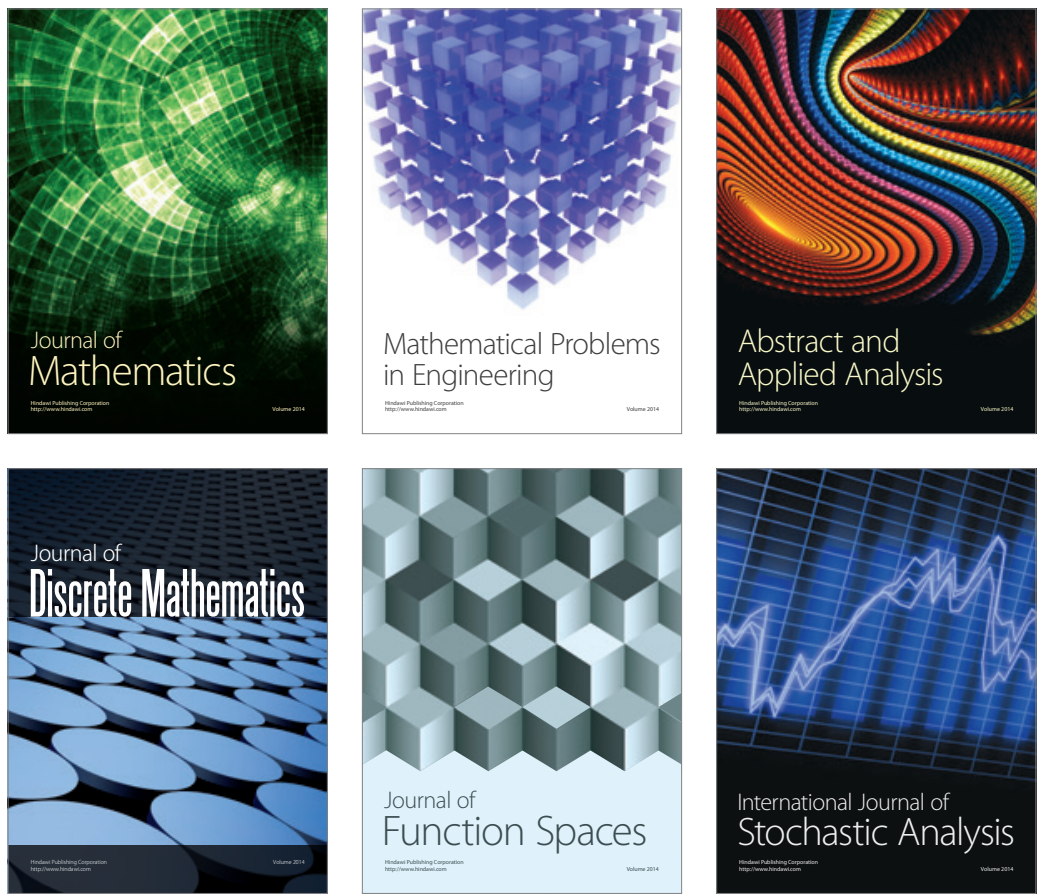

Journal of

Function Spaces

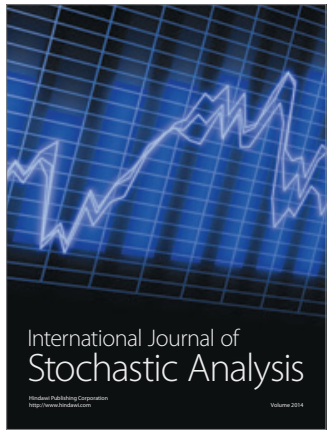

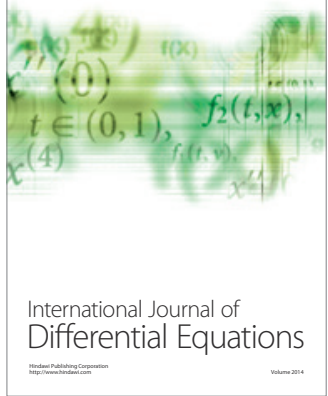
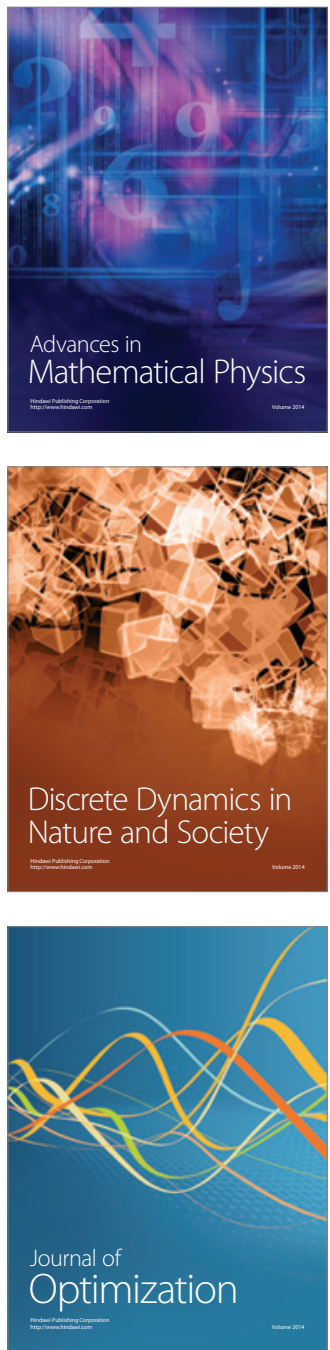